\title{
Une lettre de l'humaniste Anna Maria van Schurman (1607-1678) sur l'accès des femmes au savoir
}

\section{Barbara BULCKAERT}

\section{CpenEdition}

Journals

Édition électronique

URL : https://journals.openedition.org/clio/141

DOI : $10.4000 /$ clio. 141

ISSN : 1777-5299

Éditeur

Belin

Édition imprimée

Date de publication : 1 avril 2001

Pagination : 167-183

ISBN : 2-85816-577-7

ISSN : 1252-7017

Référence électronique

Barbara BULCKAERT, « Une lettre de I'humaniste Anna Maria van Schurman (1607-1678) sur l'accès des femmes au savoir », Clio. Histoire, femmes et sociétés [En ligne], 13| 2001, mis en ligne le 14 novembre 2006, consulté le 22 avril 2022. URL : http://journals.openedition.org/clio/141 ; DOI : https://doi.org/10.4000/clio.141

Ce document a été généré automatiquement le 22 avril 2022.

Tous droits réservés 


\title{
Une lettre de l'humaniste Anna Maria van Schurman (1607-1678) sur l'accès des femmes au savoir
}

\author{
Barbara BULCKAERT
}

[We women] may as well read in our Closets, as Men in their Colleges ${ }^{1}$.

1 Apprendre à lire et écrire, un peu de calcul, mais avant tout du catéchisme; les jeunes garçons et filles de la Renaissance ne pouvaient espérer plus de leurs écoles villageoises qui ne possédaient que peu de moyens. Si la famille était capable de payer un précepteur ou si un des parents avait un degré de formation assez élevé pour instruire ses enfants - ce qui était rare - le fils au moins avait quelques chances de recevoir une meilleure éducation. Après sa formation à domicile, le jeune homme qui avait des ambitions académiques était prêt à entrer à l'université ou dans une école supérieure. La mère se chargeait généralement de l'éducation de sa fille qu'elle initiait aux tâches domestiques ${ }^{2}$. Celles qui pouvaient assister aux cours de leurs frères ne constituaient qu'une minorité.

2 Après une éventuelle instruction à domicile, la seule façon pour la jeune femme studieuse d'atteindre un niveau académique était l'autodidaxie ${ }^{3}$, souvent sous l'œil vigilant d'un mentor, qui l'encourageait dans ses études et qui jouait également un rôle capital dans les contacts entre l'érudite et la République des lettres. Selon Frijhoff : «l'autodidacte reconnu comme tel jouit très souvent d'un statut particulier dans le monde savant. S'il est considéré à certains égards comme inférieur aux savants ordinaires, à d'autres il paraît comme nettement supérieur $»^{4}$. Cette double approche est d'autant plus vraie pour l'érudite. D'une part son œuvre est rarement reconnue comme un travail scientifique à part entière et, d'autre part, la savante est placée sur un piédestal, et admirée comme si elle bénéficiait d'un don céleste ${ }^{5}$.

Anna Maria van Schurman : « une jeune fille instruite dans pratiquement chaque discipline ${ }^{6}$

3 Anna Maria van Schurman (1607-1678) est reconnue en son temps comme une de ces figures exceptionnelles. Eduquée avec ses trois frères par un précepteur, puis par son père, elle lit et écrit le néerlandais, sa langue maternelle, à trois et quatre ans ${ }^{7}$. Lorsqu'il 
s'aperçoit que sa fille est exceptionnellement douée, son père lui apprend les difficultés grammaticales du latin, en lisant et commentant avec elle Sénèque et l'Écriture sainte. Progressivement van Schurman devient une polyglotte hors du commun. Elle maittrise le néerlandais, le français, l'anglais, l'italien, le latin, le grec ancien et l'hébreu. Elle pratique le syrien, le maure, l'arabe et l'éthiopien. Les contributions multilinguistiques calligraphiées de sa main dans les alba amicorum ${ }^{8}$ de ses contemporains sont très recherchées.

4 L'œuvre de van Schurman et sa correspondance avec des savants contemporains témoignent de sa connaissance profonde de la métaphysique, de la logique, de la philosophie, de l'éthique, de la rhétorique, de l'astronomie, de la physique, de la botanique, de la géographie, de la médecine théorique, de l'histoire et de la politique. Aujourd'hui nous connaissons surtout l'artiste van Schurman à travers ses peintures, ses dessins et ses modelages ${ }^{9}$.

5 À 17 ans, elle adresse une lettre latine au poète néerlandais Jacob Cats (1577-1660) pour lui exprimer son admiration. Cats chante les louanges de la jeune fille, ce qui suscite l'intérêt de la République des lettres. L'enfant prodige est transformée en phénomène considération que la trop modeste van Schurman n'apprécie pas toujours. Lors de la cérémonie officielle de la fondation de l'université d'Utrecht en 1636, van Schurman, considérée comme la meilleure latiniste d'Utrecht, est invitée à écrire un poème latin. Grâce à son talent extraordinaire, Anna Maria van Schurman obtient l'autorisation exceptionnelle de suivre des cours universitaires aux Pays-Bas. Dans l'auditorium, on construit une loge en bois avec quelques ouvertures afin qu'elle puisse suivre les discussions entre professeurs et étudiants sans y participer activement et sans être vue.

6 En tant qu'autodidacte, van Schurman poursuit ses études dans de multiples domaines, même si au cours de sa vie elle se concentre davantage à l'étude et à l'analyse de l'Écriture sainte et de la théologie ainsi qu'à ses sciences auxiliaires. À un âge plus avancé, Anna Maria rencontre Jean de Labadie (1610-1674), calviniste sectaire, à la tête d'une communauté religieuse. Elle le rejoint et devient à ses côtés codirigeante de la communauté qui se retire dans un château en Frise. Elle y écrit son autobiographie, menant une vie ascétique loin des mondanités de la République des lettres. Suivant l'exemple de saint Augustin (354-430), Anna Maria van Schurman révoque alors toute son œuvre antérieure.

La correspondance d'Anna Maria van Schurman : Que ceci soit donc notre thèse : l'étude des lettres convient à la femme chrétienne.

7 Dans la correspondance entre van Schurman et le théologien français André Rivet $(1572-1651)^{10}$, nous découvrons une jeune femme qui se révolte contre un système privant le sexe féminin de connaissance et de savoir ${ }^{11}$. Van Schurman a pris en effet conscience de sa situation privilégiée : fille de parents aisés, sans la responsabilité d'un ménage à plein temps et consacrant la plus grande partie de ses journées à l'étude. La lettre que nous présentons, datée du 6 novembre $1637^{12}$, fait partie d'une série de cinq lettres (trois de van Schurman à Rivet, et deux de Rivet à van Schurman, écrites entre 1632 et 1638, elles furent publiées ensemble pour la première fois en 1641) ${ }^{13}$. André Rivet est pour van Schurman un second père et interlocuteur privilégié, mais son opinion concernant la cause féminine est loin d'être progressiste :

il est prouvé maintenant et pour le futur que ce n'est pas par faiblesse mentale ou

de jugement que la plupart des femmes ne s'occupent pas de ce genre de choses

[c'est à dire les lettres et les beaux arts], mais parce qu'elles ne veulent pas y prêter 
attention, et d'autant plus qu'il n'est pas convenable que beaucoup de femmes choisissent ce mode de vie. Il suffit que quelques-unes, appelées par un instinct spécial, excellent parfois..."

Dans sa réponse, van Schurman manifeste pour la première fois ses positions «féministes ». L'Utrechtoise les énonce avec force : certaines femmes doivent avoir le droit et même le devoir de s'éduquer. Écrivant cela, van Schurman pense aux jeunes filles célibataires de la bonne bourgeoisie, ainsi qu'aux femmes n'ayant pas de charges domestiques et aux veuves ${ }^{15}$. André Rivet exprime ce que pense une grande partie des intellectuels de son temps. Il est évident que la femme a des capacités intellectuelles, mais celles-ci sont inférieures à celles de l'homme. Une femme savante doit rester une exception, parce que Dieu a prévu d'autres occupations pour elle :

Vous dites que ce n'est pas la faculté qui manque aux femmes, mais la volonté, ce qui arrive aussi chez les hommes. Je ne nie pas cela, mais pourtant, si le comportement et les études suivent le tempérament du corps, il est certain que le créateur de la nature a formé les sexes différents de telle manière qu'il entendait destiner les hommes à d'autres choses que les femmes. ${ }^{10}$

Van Schurman s'oppose à cette opinion généralement répandue qui est, selon elle, basée sur des habitudes qui se sont institutionnalisées, et non sur la raison. Ainsi l'autorité qui impose ce sort et ces limitations au sexe féminin est-elle divine ou humaine $?^{17}$ Van Schurman ne parviendra pas à convaincre André Rivet qui considère qu'elle fait une erreur de jugement en voulant généraliser son cas. Il est inutile qu'une femme fasse des études approfondies étant-donné qu'elle n'a pas le droit de mettre sa connaissance en pratique. Elle ne joue aucun rôle dans la vie publique, n'assiste jamais aux affaires politiques et ecclésiastiques et elle ne donnera certainement jamais de cours publics ${ }^{18}$.

10 À première vue l'Amica dissertatio [Discussion amicale] entre van Schurman et Rivet se termine en 1638. Trois ans plus tard, en 1641, la Dissertatio de ingenii muliebris ad doctrinam et meliores litteras aptitudine. Accedunt quaedam epistolae, eiusdem argumenti [Dissertation sur l'aptitude de l'esprit féminin pour l'étude et les belles lettres. Àccompagnée de quelques lettres sur le même sujet] est publiée à Leyde, à l'instigation de Johan van Beverwijck. Cette dissertation de van Schurman est partiellement basée sur ses lettres et défend ardemment le droit à l'étude pour les femmes. Le traité est écrit sous la forme d'une quaestio, un système logique moyenâgeux utilisé en tant que disputatio académique ${ }^{19}$. Van Schurman reprend sa thèse, avec une argumentation plus élaborée sous forme de syllogismes.

11 Selon van Schurman, la femme d'intelligence moyenne est aussi capable qu'un homme du même niveau d'entreprendre des études. L'Utrechtoise ajoute que beaucoup d'hommes de lettres n'ont en définitive qu'une intelligence moyenne. Les femmes chargées de lourdes occupations familiales ne peuvent pas se libérer, mais ceci ne vaut pas pour les jeunes filles et les dames d'un certain âge, et pour celles qui ont les moyens financiers de se faire assister dans le ménage.

12 À quelles sciences, lettres et beaux arts le sexe féminin doit-il avoir accès selon Schurman? À toutes les disciplines honorables, selon leur importance pour la compréhension de l'Écriture sainte, cela dépendant du talent de la femme. Les sciences auxiliaires de la théologie et de la morale occupent la première place : la grammaire, la logique et la rhétorique, ensuite la physique, la métaphysique, l'histoire et les langues (en particulier le grec et l'hébreu), etc. En dernier lieu viennent les mathématiques 
(dont la musique fait partie), la poésie, la peinture et les disciplines apparentées. La jurisprudence, la stratégie militaire, l'art de tenir un discours à l'université, de plaider ou de prêcher et la politique sont d'importance inférieure, mais pourtant la femme ne peut pas être exclue de l'étude de la théorie ${ }^{20}$.

L'homme aussi bien que la femme doivent s'épanouir intellectuellement, et aspirer à une meilleure connaissance et compréhension de Dieu et des actes divins. Chacun doit vouer son temps libre à des occupations nobles et élevées, et non pas à des activités superficielles, comme par exemple la parure. S'appuyant sur des témoignages et des exemples de femmes et hommes illustres du passé, van Schurman démontre que l'étude rend chaque être humain meilleur. Elle conclut sa dissertation en incitant les parents à bien éduquer leurs enfants dès le plus jeune âge.

Influence d'Anna Maria van Schurman

Nous connaissons cinq éditions de la Dissertatio ou Problema practicum [Problème pratique], manière dont le traité est désigné dans les quatre éditions des Opuscula ${ }^{21}$ [Petites œuvres] de van Schurman, qui recueillent et regroupent des lettres, quelques dissertations, des poèmes et éloges de et à van Schurman. En 1659 apparaît à Londres une traduction anglaise: The learned maid; or whether a maid may be a scholar? A Logick exercise ; Written in Latine by that incomparable virgin Anna Maria à Schurman of Utrecht. With some epistles to the famous Gassendus and others. Les Opuscula ont connu un succès immense pendant la vie de van Schurman et longtemps après (comme en témoigne la dernière édition - connue - datant du xviiie siècle). Van Beek remarque à juste titre qu'à ce jour nous trouvons encore des centaines d'exemplaires des Opuscula à travers le monde entier. Deux des éditions étaient publiées par Elsevier, éditeur qui possédaient des réseaux de distribution dans toute l'Europe ${ }^{22}$.

D'autres érudites en Europe à la Renaissance ont publié sur le statut de la femme, mais rares sont celles qui ont acquis la même notoriété que van Schurman. Les plus connues sont sans doute Le nobiltà et eccellenze delle Donne et i difetti e mancamanti de gli Huomini de l'italienne Lucrezia Marinella (1571-1653) et Égalité des hommes et des femmes de la française Marie de Gournay (1565-1645) ${ }^{23}$ - deux œuvres que van Schurman avait d'ailleurs lues. Son commentaire à leur propos est néanmoins ambigu :

Ceci est selon moi tellement loin de convenir à la modestie d'une jeune fille ou à ma pudeur innée, que j'ai même honte de lire le par ailleurs excellent traité de Lucretia Marinella, qui porte le titre 'Le nobiltà et eccellenze delle Donne et i difetti e mancamanti de gli Huomini'. Bien que je ne puisse désapprouver la petite dissertation de la très noble de Gournay, 'Egalité des hommes et des femmes', en raison de son élégance et sa subtilité, j'en n'oserai ni voudrai pourtant approuver la totalité."

Dans les Opuscula nous lisons pourtant un éloge de van Schurman à la Française : «à l'héroïne de Gournay, dotée d'une grandeur et excellence d'âme, qui défend avec ardeur la cause de notre sexe $»^{25}$.

Le réseau humaniste féminin

$17 \mathrm{Au}$ sein de la République des lettres humaniste - véritable réseau d'hommes de lettres et de sciences qui échangent informations et connaissances à travers l'Europe par le biais de contacts et de correspondances - les femmes ne sont pas absentes. Si le réseau presque entièrement masculin est de loin le plus important quantitativement ${ }^{26}$, il existe aussi des réseaux mixtes - comme l'illustre la correspondance théologique entre van Schurman, André Rivet, le théologien Gijsbert Voetius (1589-1676), le médecin Johan van Beverwijck (1594-1647) et Elisabeth, princesse de Bohème (1616-1680) - et le réseau 
humaniste féminin ${ }^{27}$. Celui-ci fonctionne selon les mêmes codes que le réseau masculin. La langue véhiculaire est généralement, mais pas nécessairement, le latin, le grec ou l'hébreu. Il n'est pas limité aux frontières des États et il s'agit de personnes qui n'ont pas nécessairement la même culture (pour ce qui s'agit de la religion, nationalité, etc.), mais qui ont des intérêts communs. Ainsi, Van Schurman entretient une correspondance avec, entre autres, la Française de Gournay, avec l'Allemande Elisabeth de Bohème, l'Irlandaise Dorothea Moore (163?-1664), et la Danoise Birgitte Thott (1610-1662) et elle accueille Christine de Suède (1626-1689) dans sa maison à Utrecht ${ }^{28}$.

Les correspondantes humanistes se soutiennent dans leurs études littéraires et scientifiques, elles se fournissent des conseils littéraires et commentent leur œuvre mutuellement. Étant donné qu'une grande partie de ces femmes se heurtaient à de vives oppositions ou étaient l'objet de mépris, parfois même dans leur entourage direct, nous estimons que ces contacts réciproques constituent non seulement un encouragement intellectuel, mais aussi social.

19 En lisant les lettres de ces humanistes, on découvre des femmes talentueuses, polyglottes, érudites dans plusieurs disciplines, qui élaborent souvent une œuvre tout aussi riche que celle de leurs pendants masculins. L'édition de l'ensemble de ces textes permettra d'affiner et de compléter cette figure de l'intellectuel à l'époque des temps nouveaux, jusqu'ici établie essentiellement à partir d'exemples exclusivement masculins.

Lettre d'Anna Maria van Schurman à André Rivet ${ }^{29}$

Je salue le seigneur André Rivet, homme très estimé.

$(. . .)^{30}$

22 J'ai reçu avec plaisir, comme il se doit, les livres avec lesquels vous désirez embellir ma bibliothèque. Ce présent m'est très agréable, tant si je me représente l'homme derrière le donateur, que le présent lui-même, source de votre triomphe. Que puis-je répondre? Je ne possède rien avec quoi je pourrais vous rendre la pareille, même si je le voulais à tout prix. À moins que l'on dise que celui qui montre sa gratitude a donné à son tour. De plus, je ne considère pas moins comme une faveur, que vous daignez de me promettre de m'aider, non seulement dans mes études, mais aussi dans la résolution de mes problèmes. Je tiens en grande valeur, dans la mesure qui sied, votre jugement, parce que quand ma connaissance est insuffisante je suis déchirée par le doute, et me vois obligée de continuer en hésitant.

Depuis tout un temps, je désire que mon opinion soit confirmée par la vôtre dans une cause qui n'est pas superficielle (parce qu'elle touche fort au devoir et à la condition des filles) et je ne considère rien de plus distingué et valeureux que votre sentiment, et pour le dire ouvertement votre réponse écrite. Au cas où vous pensez différemment, je n'aurais pas honte de sonner la retraite, après avoir été convaincue du contraire de mon opinion. La lettre que vous m'avez fait parvenir jadis, me porte néanmoins à hésiter quant à votre opinion en général sur le sujet. Après que vous vous étiez, selon votre habitude, exprimé d'une façon gentille et flatteuse concernant mes études, vous écrivez ceci : il n'y a pas davantage à ce que beaucoup de femmes choisissent ce genre de vie ${ }^{31}$; il suffit que quelques-unes, appelées par une inspiration spéciale, brillent. Si nous entendons par ceci, des ménagères engagées dans des affaires domestiques, ou n'importe quelle autre femme qui prend soin de sa famille, alors je suis immédiatement d'accord. Mais si nous parlons de filles qui sont intellectuellement prédisposées et qui 
doivent recevoir une éducation libérale et il y en a beaucoup actuellement - j'ai plus de difficulté à consentir.

Une énorme admiration pour les sciences, ou pour l'équité du droit commun, me pousse à ne pas admettre que ce qui est un désir honorable pour tous, soit une exception pour notre sexe. Et puisque la connaissance est un tel grand trésor pour l'humanité, qu'elle doit justement se répandre pour l'usage public, mais également individuel (dans la mesure du possible pour la situation de chacun), je ne vois pas pourquoi cette parure ${ }^{32}$, qui est le plus resplendissant, ne sied pas à une jeune fille que nous laissons s'embellir et se rendre attrayante.

Il n'y a aucune raison pour laquelle la république devrait craindre ce changement, vu que la gloire de l'ordre littéraire ne détruit pas la splendeur de l'ordre du pouvoir. Au contraire, chacun partage l'opinion que l'État le plus prospère sera celui dont la majorité n'est pas soumise aux lois, mais se laisse guider par la sagesse. Ajoutez à cela que tant pour la vertu, que pour le chœur des érudits, ni leur honorabilité et ni leur dignité ne peut exister quand la plupart d'entre eux sont incapable de saisir la bienséance et la splendeur des belles lettres qu'ils admirent aveuglement au lieu de les estimer avec justesse.

Mais je ne vais pas demeurer plus longtemps dans le propylée - entamons le vif du sujet. Après que celui-ci sera défini clairement, la vérité éclatera au grand jour. La question principale est la suivante : l'étude des lettres et des arts convient-elle à une jeune fille, et plus particulièrement à une jeune fille aujourd'hui ? Persuadée cependant par des preuves nullement superficielles, j'opte plutôt pour une réponse positive. Afin d'engager mon débat à partir du droit civil, je me souviens d'avoir lu que, selon Ulpien, les femmes étaient tenues à l'écart de toutes manifestations civiles et publiques. Sur quel principe d'équité cela repose-t-il ? Je ne vais pas examiner cela de manière approfondie. Je pense néanmoins que par ce point de vue il est clairement démontré que notre loisiri ${ }^{33}$ est justifié et honorable. D'où découle pour nous forcément beaucoup de temps et de repos favorable aux muses - surtout si par un avantage spécifique nous sommes libérées des occupations nécessaires et dispensées des soucis et des obligations domestiques. Mais bien sûr quand ce temps libre s'écoule en débauche et négligence et n'est pas employé à quelque chose d'utile, alors s'ouvre le chemin de tous les vices. Saint Basile l'a bien exprimé : la paresse est le premier des vices. Car comment éviter cette Charybde, si l'esprit n'est pas alerté, ne s'amollit-il pas et ne commence-t-il pas à ressembler à l'inutilité et la paresse, dans lesquelles il se complait? Quoi alors? Écoutons Sénèque, cultivateur des esprits élevés, qui ouvre pour nous le chemin entre les écueils. Puisque, dit-il, seuls ceux-là sont libres (c'est-à-dire, qui occupent au maximum leur temps libre), ceux qui ont du temps pour la sagesse ; ils sont les seuls qui vivent. Ils n'occupent pas seulement bien leur propre temps, ils y ajoutent tous les temps. Nous ne devons d'ailleurs pas chercher le temps libre loin des bonnes choses, mais les meilleures choses dans le temps libre. Ainsi, une meilleure sérénité dans l'isolement, ne provoquera ni inconvénient, ni ennui. Puisque (selon Cicéron) il y a deux choses qui mènent à la langueur des autres, mais qui inspirent les savants, notamment le temps libre et la solitude.

Mais il y en a qui suggèrent que le filage et la couture suffisent à la femme. Je reconnais cependant que beaucoup de gens en sont persuadés et que des personnes malveillantes aujourd'hui les suivent généralement. Mais nous n'acceptons pas cette règle de Lesbos ${ }^{34}$ - nous recherchons la voix de la raison, pas celle de l'habitude. De quel droit, je vous le 
demande, ce sort nous accablerait-il ? Divin ou humain? Jamais ils ne prouveront que ces restrictions seraient notre sort, ou qu'elles soient prescrites par le ciel, par quoi nous sommes bien entendu poussées dans les rangs. Parce que si nous cherchons un témoignage dans l'Antiquité, alors les exemples de tous les temps, et même l'autorité des hommes les plus importants prouvent le contraire. Ainsi la très honorable Marie de Gournay ${ }^{35}$ le démontre dans son petit livre qu'elle intitulait L'égalité des hommes et des femmes.

Mais pour que je ne reprenne pas les choses du passé, comme on dit, j'éviterai de les réexaminer. Il me suffit d'y ajouter avec un raisonnement clair, que les choses les plus élevées non seulement nous conviennent, mais nous attendent dans ce genre de vie. Et quelqu'un pourvu d'une noble nature, ne tolère pas d'être confiné dans ses frontières, ni que la perspicacité de son esprit développé soit oppressée et réduite. Au cas où les lois draconiennes doivent être maintenues, je ne m'étonne pas tellement que certaines femmes soient parfois entraînées dans les tentations d'un monde séduisant, par mépris d'une tâche indigne. À cela s'ajoute qu'il ne nous reste aucun espoir d'honneur, de dignité ou de quelque récompense par quoi les âmes non corrompues sont d'habitude incitées le plus à des actes louables. En vain, nous nous glorifions pour ainsi dire de notre noblesse, héritée de nos prédécesseurs, étant donné qu'elle s'égare sans profit dans l'obscurité. C'est ainsi que pour celui qui lira l'histoire, le souvenir ancien de notre nom se perd comme l'écume d'un bateau qui traverse la mer. Mais, demanderont-ils; où est votre honneur? Qu'est-ce qui vous rend immortelles? Pas votre loisir tout de même ? Pourquoi pas? Mais de ce temps libre qui brille par la lumière de la science. Il nous convient de devenir célèbre grâce à Pallas sans son armure, mais bien plutôt en toge. Quand la véritable philosophie occupe le trône de notre pensée il n'y a plus d'accès pour les agitations inconstantes d'un esprit tourmenté. Érasme, protecteur des belles lettres rendait cela avec éloquence, quand il parlait de l'éducation des filles de Thomas More : Nulle chose, dit-il, comme l'étude ne remplit autant le cœur d'une jeune fille ${ }^{36}$.

29 Comment ne pas refuser sans danger le luxe de ce monde, les mauvais exemples dont l'attrait force l'adhésion et la frivolité de ce temps effréné, quand des sommets de la science nous regardons les choses terrestres ? De plus, puisque le désir de perfectionner nos origines (ce que personne de nous ne manque de faire) concerne moins notre devoir que notre bonheur à tous, nous devons réaliser que l'image royale de celui qui est lumière et vérité, brille de plus en plus au profond de notre âme. Néanmoins, je ne nie pas que la théologie (puisqu'elle perfectionne le mieux l'esprit) remplit les deux rôles. Pourtant ceux qui veulent qu'elle fasse son entrée toute seule et sans ses aides, paraissent ne pas assez approfondir la majesté d'une telle reine ${ }^{37}$.

Et quand nous regardons un livre de sciences naturelles, qui ne voit pas comment les parties de ces deux sciences se complètent harmonieusement, combien se complètentils avec soutien ou clarté. Que certains veuillent garder cette étude dans de telles frontières rigoureuses, ne doit pas nous retenir. Ils estiment que cela n'a pas d'importance pour nous que cette construction terrestre est constituée d'atomes; ou est née de chaos sans forme; ou que certains éléments possèdent par hasard des qualités divines ou terrestres; ou que la masse supérieure de la terre tourne en rond; ou que ceux qui prétendent que ce n'est pas la masse supérieure qui tourne mais l'inférieure ont des problèmes avec la rotation; ou que le soleil couchant coule dans 
l'océan ; ou que sa lumière sert également aux antipodes ; ou que la terre est ronde ou carrée ; ou finalement que tout l'univers s'arrête à l'horizon, ou à notre acuité visuelle.

Si nous devions écouter de tels discours, qui sont en général prononcés en vue de notre déshonneur, alors nous accepterions aussi que Dieu, créateur de l'univers, qui nous conduisit à ce théâtre afin de connaître et d'honorer ses plus belles œuvres, serait déçu dans ses résolutions par nous. Parce que la nature n'est pas pour nous une telle marâtre, qu'elle nous aurait refusé de l'admirer. Sinon, pourquoi nous aurait-elle donné ce qui est en chacun de nous selon le philosophe, c'est à dire le désir d'apprendre? Pourquoi nous aurait-elle permis de nous tenir debout, si ce n'est pour diriger également nos yeux et notre esprit dans sa direction.

En tout cas, nous serions des idiots, et non des êtres humains, des étrangers, et non des habitants de ce monde, si nous ne dirigions pas notre attention, excitée et enflammée comme par l'amour divin, vers ces belles et hautes sphères, dans lesquelles brille la majesté divine. Il n'y a pas de raison de penser que nous faisons parfaitement notre devoir, en regardant quelques fois ces choses par une fenêtre munis de barreaux. Parce qu'ainsi nous ne regardons pas afin de connaitre, mais par ce fait même nous montrons que nous n'explorons pas au-delà de ce que nous ne voulons pas connaître. Rien de plus merveilleux ne se présente devant nos yeux que l'être humain, rien de plus beau que le siège de l'âme. Mais comment est petit quelque chose qui est jugé selon sa peau ou son image extérieure. Combien nous devons nous gêner par rapport aux excellents hymnes de ces païens dans lesquels ils chantent l'honneur du créateur supérieur, pendant qu'ils examinent la nature d'une façon profonde, et par cette recherche approchent la première cause de tout. En plus, chaque fois que nous tournons notre attention vers la gloire des Écritures saintes, nous trouvons tant d'exemples de saintes personnes, qui par l'étude de cette matière ont décidé de célébrer leur Dieu que, comment ne serionsnous pas obligés de montrer la même gratitude.

Mais, pour ne pas parler trop en général de l'étude de l'histoire, qui aujourd'hui se maintient dans les gynécées et à la cour des grands, nous examinerons uniquement en passant si la connaissance des affaires publiques convient à chaque personne privée. En ce qui concerne la pratique et le fonctionnement de la république, je concède facilement que cela sert d'une façon directe. Or nous estimons que personne ne peut la négliger à cause de la théorie et des avantages personnels qui en découlent pour chacun. Ici également les livres saints nous précèdent et non seulement ils nous précèdent, mais ils nous y guident en nous prenant par la main. Là, le fil des temps est composé par les périodes de gouvernement ; là, on décrit ou annonce la naissance aussi bien que la destruction des grands peuples. Ceci est peu étonnant, étant donné qu'ici, les jugements stupéfiants de Dieu, que nous devons toujours respecter, apparaissent dans une forme splendide; et que tout cela ne peut arriver dans la vie d'un humain.

Si Dieu avait voulu que cette matière touche le cœur de tout le monde, la contemplation de ce règne admirable n'aurait-elle pas également animé nos cithares? Le musicien royal s'y consacre de tout cœur et s'exclame souvent : Ô Seigneur, que vos pensées sont profondes. Quelqu'un pourrait peut-être objecter que ceci n'est autre que la recommandation de la vie monastique ou la définition par seule spéculation des limites de notre devoir. Mais la raison semble exiger que nous faisons d'abord assez attention à nous-mêmes, pour trouver notre propre bonheur, et après à notre prochain. Celui qui n'a pas suffisamment de loisir pour soi, libère vainement son temps pour les autres. Il est inutile que quelqu'un qui ne sait pas prendre soin de lui/elle-même, apporte aide et 
conseil à autrui. Et finalement, celui qui est étranger dans sa propre maison aspire en vain à des rapports avec des citoyens ou à un lien avec la communauté chrétienne plus élevée.

Quelle témérité, je vous prie, que de vouloir bâtir l'autorité de nos valeurs morales sur une base d'ignorance ou sur l'opinion publique. Si, par contre, on excluait le hasard, les exemples de toutes les époques nous apprennent que, si quelqu'un est instruit par la science grande et vraie, personne ne sacrifie avec d'heureux présages aux dieux si grands. Le savoir, en effet, nous prépare, nous dispose et nous assigne à bien agir, et stimule notre esprit à accomplir des actes plus admirables. Rien n'est plus utile et plus nécessaire pour une jeune fille que de savoir discerner la honte de l'honneur, le nuisible de l'inoffensif et l'indécence de la décence. Combien d'expérience en matière, combien de dextérité de jugement cela ne nécessite-t-il pas? Mais, vu qu'il n'est pas à conseiller, en qu'il n'est pas suffisamment sûr d'acquérir cette connaissance par sa propre expérience, nous devons faire appel à l'histoire. Ceci est nettement démontré par la citation suivante : pour, comme dans un miroir, embellir et imiter la vie par les vertus des autres.

Je vous dis que nous nous trouvons dans l'impossibilité de regagner les bonnes grâces avec une bonne renommée, quand la souillure d'une suspicion triviale commence à s'y attacher. D'ailleurs, les jeunes filles se soucient beaucoup à, non seulement écarter le mal après s'y être exposées, mais surtout à le prévenir, pour ne jamais y être exposées.

Enfin, pour passer sous silence les arts et les sciences auxiliaires (comme elles sont appelées), car elles suivent forcément les sciences principales comme des servantes suivent leur reine, je dois prendre la parole à propos de la joie que nous éprouvons à être polyglotte, non pour la parure, mais pour l'utilité. Car les langues sont les gardiennes de la foi, ou plutôt les vecteurs de la transmission de l'antiquité sage. Quand elle nous parle dans son propre idiome, elle nous montre une image fidèle de son âme et elle affecte nos sens de sa merveilleuse grâce et de son charme - ce que nous réclamons à juste titre pour toute traduction, aussi excellente qu'elle soit. Si je voulais vous expliquer combien il est agréable et fertile de puiser et d'interpréter la doctrine céleste à partir de ses sources mêmes, cela ne serait en rien différent de ce que dit le proverbe : prêter de la lumière au soleil.

Mais pour conclure, voici un exemple qui me vient sans cesse à l'esprit. Un exemple, je vous dis, de l'incomparable princesse Jane $\mathrm{Grey}^{38}$. Aucun peuple, aucune époque (si tout le monde me le permet) ne trouvera son pareil. Le Florentin Michelangelo, qui écrit de façon passionnée l'histoire complète de sa vie et de sa mort sous la forme d'un dialogue entre elle-même et Feckenham, qui annonça sa mort approchante, nota entre autres ceci : elle n'estimait guère ce qu'elle reçut en outre de Dieu, en talents, sang noble, beauté, jeunesse de la fleur de l'âge, grâce auxquels elle aurait pu se procurer la gloire et la reconnaissance de ce monde. Pleine de conviction elle proclame que dans toute sa vie rien ne lui était aussi cher que sa connaissance des trois langues, que l'on nomme les langues des savants. Et si le plaisir et le divertissement qui nous échoit dans cette vie, nous doit atteindre au nom du véritable bonheur, elle admet qu'elle trouva sa félicité dans l'étude des belles lettres et surtout dans la sainte Écriture. Bien que beaucoup de personnes critiquent ce genre d'études pour une femme, elle y avait néanmoins trouvé beaucoup de consolation spirituelle, qu'elle avait ressentie, et qu'elle ressentait encore toujours en son intérieur. Elle jugea que leur opinion allait à l'encontre de toute raison. 


\section{BIBLIOGRAPHIE}

Agrippa Henri Corneille, 1990, De nobilitate et praecellentia foeminei sexus. Edition critique d'après le texte d'Anvers 1529. Préface de R. Antonioli, établissement du texte par Ch. Béné, traduction de $\mathrm{O}$. Sauvage, notes de R. Antonioli, Ch. Béné, M. Reulos, O. Sauvage, Genève.

Bulckaert Barbara, 1995, «Vrouw en eruditie : het 'Problema Practicum' van Anna Maria van Schurman (1607-1678) », Miscellanea Jean-Pierre Vanden Branden, Archives et bibliothèques de Belgique, $n^{\circ}$ 49, Brussel, pp. 145-195.

--, 1997, « L'éducation de la femme dans la correspondance d'Anna Maria van Schurman (1607-1678) et André Rivet (1572-1651) », in M. Bastiaensen (ed.), La femme lettrée à la Renaissance, Travaux de l'Institut Interuniversitaire pour l'Étude de la Renaissance et de l'Humanisme, Actes du Colloque international, Bruxelles, 27-29 mars 1996, n XII, Leuven, pp. 197-209.

A collection of seventy four letters and four Latin poems etc. in the handwriting of the very talented and very celebrated Anna Maria de Schurman, the letters almost altogether addressed to Andreus Rivet D.D., tutor of the young Prince of Orange and author of 'Critici Sacri' etc. etc., 1632-1669, La Haye, Koninklijke Bibliotheek, Mss., 133B8. (dans le texte : La Haye, KB, Mss., 133B8).

Frijhoff Willem, 1983, «Wetenschap, beroep en status ten tijde van de republiek : de intellectueel ", Tijdschrift voor de geschiedenis der geneeskunde, natuurwetenschappen, wiskunde en techniek, 5, pp. 18-30.

--, 1996, « Autodidaxies, xvie-xixe siècles : jalons pour la construction d'un objet historique », Autodidaxies XVIe-XIXe siècles, Paris, W. Frijhoff (ed.).

A History of Women Philosophers,1600-1900, 1991, vol. 3, Dordrecht/Boston/London, M.E. Waithe (ed.).

Jardine Lisa, 1985, « O Decus Italiae Virgo' or the Myth of the Learned Lady in the Renaissance », The Historical Journal, 28-4, pp. 799-820.

Kelso Ruth, 1956, Doctrine for the Lady of the Renaissance, Urbana. 
Van Beek Pieta, 1998, « De 'Opuscula Hebraea Graeca Latina et Gallica prosaica et metrica' van Anna Maria van Schurman (1607-1678) als boek », Tydskrif vir Nederlands en Afrikaans, 5-1, pp. 5373.

Van der Stighelen Katlijne, 1987, Anna Maria van Schurman of 'Hoe hooge dat een maeght kan in de konsten stijgen', Leuven.

Van Schurman Anna Maria, 1673, Eukleria seu melioris partis electio. Tractatus brevem vitae eius delineationem exhibens, Altona.

,-- 1685 , Eukleria seu melioris partis electio. Pars secunda, historiam vitae eius usque ad mortem persequens, Amsterdam.

Vives Juan Luis, 1996, De institutione feminae christianae, liber primus. Introduction, critical edition, translation and notes, Leiden/New York/Köln, C. Fantazzi et C. Matheeussen (edd.).

\section{NOTES}

1.Paroles célèbres de la philosophe Margaret Cavendish (1623-1673). Voir : Waithe (ed.) $1991: 1$.

2.L'humaniste espagnol Vives écrit un ouvrage de base sur l'éducation féminine, qui aura une très grande influence à travers toute la Renaissance. Voir : Vives 1996 : 12-52. - Kelso 1956 : 38-57.

3.Très peu de recherches ont été effectuées sur l'autodidaxie à l'époque moderne, et les rares études excluent en général l'érudite, bien que toute femme lettrée soit forcément autodidacte!

4.Frijhoff $1996: 18$.

5.Jardine 1985.

6.Ceci sont les paroles traduites du latin de Friedrich Spanheim (1600-1649) dans la préface au lecteur, dans : van Schurman 1652 : I-II. Pour plus de données biographiques sur Anna Maria van Schurman je recommande : Van der Stighelen 1987. - van Schurman 1673. - van Schurman 1685. À ma connaissance il n'existe pas de biographie étendue en français.

7.Van Schurman réside la plus grande partie de sa vie à Utrecht.

8.Des livres d'amis, comparable à des albums pour poésies que les humanistes s'échangeaient.

9.Touchant l'artiste van Schurman je renvoie à : Van der Stighelen 1987.

10.Bulckaert 1995 : 164.

11.Bulckaert 1997.

12.Pour la traduction et la date de la lettre nous suivons la leçon de l'autographe (référence : La Haye, KB, Mss., 133B8, n 14). Sous sa forme publiée la lettre porte la date du 8 mars 1638.

13.Trois des cinq lettres étaient publiées auparavant, sous le titre Amica dissertatio inter nobilissimam virginem Annam Mariam a Schurman et Andraeam Rivetum, de ingenii muliebris ad scientias, et meliores litteras capacitate [Dissertation amicale entre la très noble jeune fille Anna Maria van Schurman et André Rivet, sur la capacité de l'esprit féminin aux sciences et aux belles lettres] à Paris en 1638. L'Amica Dissertatio a été traduit en français par Guillaume Colletet et publié sous le titre Question célèbre s'il est nécessaire, ou non, que les filles soient sçavantes, Paris, 1646. La première publication en 1641 à Leyde du traité de van Schurman sur l'éducation féminine (voir 
plus loin) reprend les trois lettres et ajoute deux lettres sur le même sujet. Il s'agit de la Dissertatio de ingenii muliebris ad doctrinam et meliores litteras aptitudine (voir plus loin).

14.Van Schurman $1652: 56$ (lettre du 15 février 1632).

15.Dans sa lettre du 6 novembre 1637 : La Haye, KB, Mss., 133B8, $n^{\circ}$ 14. - van Schurman $1652: 65$. Dans sa dissertation : Bulckaert $1995: 163$.

16.Lettre du 18 mars 1638 : van Schurman $1652: 75$.

17.Lettre du 6 novembre 1637 : La Haye, KB, Mss., 133B8, n 14. - van Schurman 1652 :

63.

18.Rivet se réfère aux écrits de l'apôtre Paul. Dans sa lettre du 18 mars 1638 : van Schurman $1652: 78$.

19.Concernant la Dissertatio, je renvoie à : Bulckaert 1995 (introduction, édition critique avec commentaire et traduction en néerlandais) - Bulckaert 1997 : 205-208.

20.Bulckaert $1995: 178$.

21.Les éditions de 1641, 1648 et 1650 (Leyde), de 1652 (Utrecht) et 1749 (Leipzig).

22.Van Beek 1998 : 66-69.

23.La fille d'alliance de Michel Montaigne était une autodidacte pur-sang. Vu que ses parents avaient une vue très traditionnelle sur l'éducation de leur fille, de Gournay étudiait seule, après avoir fini les tâches ménagères. Elle appris le latin en comparant les textes avec leurs traductions françaises. Pourtant moins talentueuse que la brillante van Schurman, sa passion pour la cause féminine apparaît d'autant plus ardente dans son œuvre 'féministe'.

24.Dans sa lettre du 14 mars 1638 : van Schurman $1652: 85$.

25.Van Schurman 1652 : 303 (traduit du latin par nos soins).

26.Un réseau ne se caractérise pas seulement par ses membres, mais aussi par les sujets (la théologie, la médecine, l'éducation féminine, la politique, etc.) de correspondance. En général une personne fait parti de plusieurs réseaux. Le réseau autour d'Érasme, Pierre Gilles, Thomas More et Guillaume Budé est un bel exemple de ce premier type de réseaux.

27.La recherche sur les réseaux humanistes masculins n'est encore qu' à ces premiers pas, et n'existe que depuis quelques années. Les réseaux féminins sont actuellement encore rarement sujet de recherche.

28.Plusieurs lettres (en français, latin, hébreu et grec) des correspondantes de van Schurman ont été recueillies dans les Opuscula. Voir van Schurman 1652.

29.Cette lettre de van Schurman est originellement en latin, et fait partie de l'Amica dissertatio dont nous parlons dans l'introduction. Ceci est un des documents les plus importants pour la Renaissance concernant l'accès des femmes au savoir. La traduction a été effectuée par mes soins, traduit du neerlandais par Bea Van Ve Wiele, et revue par Alexandre Vanautgaerden.

30.Pour la commodité de la lecture nous avons supprimé le paragraphe suivant : « Rien de plus agréable n'aurait pu m'arriver, homme respectable et honoré père en Jésus Christ, que de remarquer, dans la mesure où ma médiocrité me permet de me consacrer à votre petite fille, que vous accueillez avec tant d'affection toute chose, aussi infime soit-elle que j'apporte à l'éducation de votre petite fille. Mais au cas ou vous aimeriez juger la chose en elle-même, à mon avis ceci était peu de choses. Car si vous considérez toutefois l'impact de notre affection, rien en tout cas ne s'est rajouté, par lequel je me semblais obligée envers vous à cause de notre ancienne amitié. »

31.Une vie de femme lettrée.

32.C'est-à-dire la connaissance. 
33.Dans la suite de la lettre van Schurman joue sur la dualité du mot latin otium, qui signifie loisir, à la fois le temps du repos, mais aussi temps consacré aux études.

34.Pendant l'Antiquité les jeunes filles grecques apprenaient des tâches ménagères et de l'ouvrage dans des écoles à l'île de Lesbos.

35.Voir l'introduction en ce qui concerne Marie de Gournay.

36.Dans une lettre à son ami Guillaume Budé (1467-1540), Érasme (1469-1536) fait les louanges de Margaret, Elisabeth et Cecily, filles de Thomas More (1478-1535), et de leur cousine, Margaret Giggs, qui recevaient une éducation intellectuelle de haut niveau à domicile. Thomas More était d'avis qu'une femme a autant besoin qu'un homme d'une bonne éducation, même si elle ne pourra pas mettre en pratique la connaissance acquise. Ses filles étaient instruites en philosophie et théologie, en plusieurs sciences, en langues (notamment le latin, grec et français) et littérature. Érasme, Ep. 1233, ad Budaeum, Allen (ed), IV : 577, r 63-77.

37.Van Schurman entend par là que l'on ne sait pas comprendre la théologie, sans maîtriser les sciences auxiliaires.

38.En 1553 Jane Grey (1537-1554) était reine d'Angleterre pendant neuf jours, avant d'être exécutée par Mary I Tudor (Bloody Mary) (1516-1558). Grey était une humaniste lettrée réputée brillante et intéressée par la philosophie. Elle maîtrisait le latin, le grec et le français.

\section{RÉSUMÉS}

Pendant la Renaissance nous observons plusieurs changements à l'égard de la femme lettrée. Bien que le sexe féminin est exclu de tout enseignement formel, les érudites revendiquent le droit à une formation intellectuelle et s'avèrent d'excellentes autodidactes. Au-delà des frontières, elles maintiennent une correspondance de haut niveau via des réseaux qui se développent entre elles. Anna Maria van Schurman (1607-1678) est une de ces humanistes qui maintient une correspondance active avec ses contemporains lettrés, et qui s'oppose au fait qu'elle est regardée comme exception en tant qu'érudite. Selon van Schurman chaque femme qui a le temps et les moyens doit se vouer à l'étude. Malgré l'absence d'un degré académique, nous pouvons sans hésitation considérer ces femmes lettrées comme des intellectuelles à part entière.

The Renaissance witnessed several changes regarding the status of the female scholar. Although the female sex was excluded from all formal education, women claimed their right to intellectual training and proved excellent autodidacts. Through international communication networks they maintained intense epistolary relationships with one another. Anna Maria van Schurman (1607-1678) was one of those humanists who corresponded actively with male and female scholars of her time. She disagreed with the idea that she should be considered exceptional because erudite. Van Schurman proclaimed that women with the time and the means should educate themselves. These female scholars of the Renaissance can be considered worthy intellectuals even though they lack academic degrees. 


\section{AUTEUR}

\section{BARBARA BULCKAERT}

Barbara Bulckaert, philologue classique, chercheuse à la Vrije Universiteit Brussel (Belgique), membre du Centrum voor vrouwenstudies de la VUB, prépare une thèse doctorale sur la position de l'intellectuelle en Europe à la Renaissance. Elle a publié « L'éducation de la femme dans la correspondance d'Anna Maria van Schurman (1607-1678) et André Rivet (1572-1651) », in M. Bastiaensen (dir.), 1997, La femme lettrée à la Renaissance, Travaux de l'Institut Interuniversitaire pour l'Étude de la Renaissance et de l'Humanisme, Leuven, pp. 197-209. 